medRxiv preprint doi: https://doi.org/10.1101/19009696; this version posted October 22, 2019. The copyright holder for this preprint (which was not certified by peer review) is the author/funder, who has granted medRxiv a license to display the preprint in perpetuity.

This article is a US Government work. It is not subject to copyright under 17 USC 105 and is also made available for use under a CCO license.

\title{
Profiles of cognitive change in preclinical Alzheimer's disease using change-point analysis
}

Owen A Williams ${ }^{\mathrm{a}}$, Yang An ${ }^{\mathrm{a}}$, Nicole M Armstrong, Melissa Kitner-Triolo ${ }^{\mathrm{a}}$, Luigi Ferrucci ${ }^{\mathrm{b}}$, Susan M Resnick ${ }^{\mathrm{a}}$

aLaboratory of Behavioral Neuroscience, National Institute on Aging, Baltimore, Maryland 21224, USA

${ }^{b}$ Longitudinal Studies Section, Translational Gerontology Branch, National Institute on Aging, Baltimore, MD, USA

\begin{abstract}
Introduction: Change-point analyses are increasingly used to identify the temporal stages of accelerated cognitive decline in the preclinical stages of Alzheimer's Disease (AD). However, statistical comparisons of change-points between specific cognitive measures have not been reported.
\end{abstract}

Methods: 165 older adults (baseline age range: 61.1-91.2) from the Baltimore Longitudinal Study of Aging developed AD during follow-up. Linear and non-linear mixed models were fit for 11 cognitive measures to determine change-points in rates of decline before AD diagnosis. Bootstrapping was used to compare the timing of change-points across cognitive measures.

Results: Change-points followed by accelerated decline ranged from 15.5 years (Card Rotations) to 1.9 years (Trail-Making A) before AD diagnosis. Accelerated decline in Card Rotations occurred significantly earlier than all other measures, including learning and memory measures.

Discussion: Results suggest that visuospatial ability, as assessed by Card Rotations, may have the greatest utility as an early predictive tool in identifying preclinical AD.

Key Words: Preclinical, Cognitive Decline, Change-point analysis, Visuospatial ability, Alzheimer's Disease 


\section{Introduction}

Alzheimer's disease (AD) is a progressive disease with a long preclinical phase in which pathological markers are present for years and even decades before clinical symptoms [1]. Decline in episodic memory is a hallmark of $\mathrm{AD}$, but other cognitive domains are also vulnerable to AD. [2] Understanding the temporal stages of the early acceleration of declines in various cognitive domains in preclinical AD is important for identifying individuals vulnerable towards accumulating $A D$ pathology and for characterizing AD progression prior to symptom onset.

The early preclinical phase of AD is characterized by beta amyloid (A $\beta)$ and phosphorylated tau accumulation with subsequent acceleration of brain atrophy in the absence of clinical symptoms. [1, 3-5] With multiple anti-amyloid clinical trials failing to show that removal of $A \beta$ is associated with improved cognitive outcomes, [6] one argument is that patients are not being targeted early enough. [6, 7] The failures of clinical trials at later disease stages have led to increasing focus on the earlier phases of disease, including the asymptomatic preclinical stage, with the hope that treatments at this stage may be more effective. [8, 9] Thus, it is critical to define the earliest, and possibly subtle, changes in cognitive performance in preclinical $\mathrm{AD}$ to identify individuals who would have the greatest potential to benefit from clinical interventions. To fully characterize cognitive changes in preclinical $\mathrm{AD}$, it is important to examine a broad range of cognitive domains and neuropsychological measures that may be sensitive to the earliest changes.

One way of investigating the timing of cognitive decline prior to clinical AD diagnosis is to use change-point analyses. Changepoint methods align participants by anchoring them at time of diagnosis to then examine trajectories of variables of interest retrospectively for timepoints of change prior to clinical diagnosis. Change-points are identified using piece-wise linear components separated by knots delineating between intervals with differing rates of change. $[10,11]$ Previous studies using change-point analyses in $\mathrm{AD}$ focused on verbal memory, $[12,13] \mathrm{re}-$ porting steeper declines in Immediate Recall, measured by the picture version of the Free and Cued Selective Reminding test, between 1 and 8.1 years before clinical diagnosis. However, memory is not the only cognitive domain subject to decline prior to $\mathrm{AD}$ onset [2] and other domains have shown early change-points. In a systematic review of change-point studies in dementia and AD, [14] the measure with the earliest changepoint, at 9.6 years before AD diagnosis, was the Block design test assessing visuospatial ability. [15] Measures associated with other domains, i.e., language fluency and executive function showed change-points detected at 6.8 years [16] and 2.9 years [13] prior to AD diagnosis respectively.

While the systematic review by Karr et al. [14] allows for a cursory comparisons of change-points between measures associated with various cognitive domains, the authors highlight various methodological differences between studies that make it difficult to draw conclusions from such comparisons. For example, the maximum length of longitudinal testing prior to AD diagnosis ranged from 930 years with frequency of visits varying between studies. Furthermore, the mean baseline ages in all studies ranged from 70-82 years, and analyses were adjusted by different sets of covariates. Therefore, the temporal sequence of changes in different cognitive domains in preclinical $\mathrm{AD}$ remains unclear.

To elucidate the temporal sequence of cognitive changes prior to clinical AD onset, we investigated changes in rates of decline on multiple cognitive measures, representing 
specific cognitive domains, in individuals who eventually developed AD. The aims of this study were to identify how many changepoints best characterize the trajectories of change in performance on several cognitive measures and to find the earliest point in time before AD diagnosis that accelerated decline in performance could first be detected for each measure.

\section{Methods}

\section{Participants}

Participants were from the Baltimore Longitudinal Study of Aging (BLSA), a longitudinal study started in 1958. [17] Participants were community-dwelling volunteers who were healthy at enrollment. During each visit, they received comprehensive psychological evaluations. For this study, we selected participants who were diagnosed with AD during follow-up and only used data from visits when they had complete neuropsychological testing data across the 11 measures investigated.

\section{Clinical diagnosis of Alzheimer's disease}

Clinical and neuropsychological data from each participant were reviewed at a consensus case conference if their clinical dementia rating score [18] was 0.5 or greater or if they had more than three errors on the Blessed Information-Memory-Concentration Test, [19] and participants were evaluated by case conference upon death or withdrawal. MCI status was determined using the Petersen criteria. [20] Diagnoses of dementia and AD were based on criteria outlined in the Diagnostic and Statistical Manual of Mental Disorders, third edition, revised [21] and the National Institute of Neurological and Communication Disorders and Stroke - Alzheimer's Disease and Related Disorders, [22] respectively.

\section{Neuropsychological measures}

Participants were administered a comprehensive battery of cognitive measures assessing verbal learning and memory, figural memory,

Table 1: Subject demographics

\begin{tabular}{ll}
\hline N Subjects & 165 \\
Total N assessments & 998 \\
Female (\%) & $83(50.3)$ \\
White/nonwhite (\%) & $141(85.5) / 24$ \\
& $(14.5)$ \\
Baseline age & $76.5(7.4)$ \\
& $61.1-91.2$ \\
Education & $16.7(2.6)$ \\
& $8-21$ \\
Age at MCI onset & $84.0(6.1)$ \\
& $65-98$ \\
Age at AD diagnosis & $86.5(6.1)$ \\
& $65-102$ \\
Follow-up interval & $8.3(6.0)$ \\
& $0-24.1$ \\
Interval between MCI & $2.5(1.8)$ \\
onset and AD & $0-10$ \\
Diagnosis & \\
\hline
\end{tabular}

attention and processing speed, executive function, language, and visuospatial ability. Here, we provide a summary of each measure used as outcomes in the present study.

The California Verbal Learning Test (CVLT) [23] assesses episodic verbal learning and memory. There are 5 learning trials of 16 shopping items, presented orally, with four items from each of four semantic categories. The sum of the five trials provides a measure of new learning and immediate free recall. In addition, short- and long-delayed free recall, short- and long-delayed cued recall, and recognition memory are assessed. The two measures used were: total number of items recalled across the five immediate free recall trials (CVLT-IMM), and long-delay free recall (CVLT-LD), with maximum scores of 80 , and 16 , respectively. 
The Benton Visual Retention Test (BVRT) [24] measures visual constructional skill and short-term figural memory. Participants study 10 line-drawings, including one to three geometric figures, for 10 seconds each, and then immediately reproduce them from memory using pencil and paper. The designs become more difficult over the 10 trials. The measure used was the total number of errors.

The Trail-Making Test Parts A (TMT-A) and B (TMT-B) [25] assess attention, concentration, visuomotor scanning, perceptuomotor speed, working memory, and set-shifting. TMT-A involves drawing a line to connect randomly arranged numbers from 1 to 25 in sequential order. TMT-B involves connecting randomly arranged numbers and letters in alternating sequence (e.g., 1-A-2-B ...). Time to completion for each test (in seconds) was used in the present study.

Letter [26] and Category [27] Fluency are measures of fluent language production and executive function. Participants were given 60 seconds to generate as many words as possible beginning with specific letters and specific categories. The mean numbers of correct words generated in 60 seconds, across the three trials each for letter and category fluency, were the measures of interest.

The Boston Naming Test (BNT) [28] is a measure of object recognition and semantic retrieval. Participants identify and name a series of line drawings of objects, beginning with common objects and ending with infrequent objects. The measure used was the number of words out of 60 correctly named without cues.

WAIS-R Similarities measures verbal concept formation and abstract reasoning. [29] Participants are asked how 14 pairs of two items are similar, starting with concrete items and becoming increasingly abstract. The measure of interest was the total score out of a maximum of 28 .
A modified version [30] of the Educational Testing Service Card Rotations test was used to measure visuospatial ability. Participants were presented with a target figure and eight alternative figures in the same row. Subjects marked images that could be rotated in plane to match the target, but not those that were mirror image figures. The total number correct minus total number incorrect across the two parts (14 targets per part) was the measure of interest, with a maximum score of 224.

The Mini Mental State Examination (MMSE) [31] assesses mental status, including orientation to time and place, immediate and delayed recall, attention and calculation, and language. Total score out of 30 was the measure of interest.

\section{Statistical analyses}

To find the number of change-points where the rate of longitudinal decline changes significantly and the timing of these changepoints relative to $\mathrm{AD}$ diagnosis, a series of linear and nonlinear mixed models with increasing complexity were fit with each of the cognitive measures as the outcome and the time (in years) to diagnosis of $\mathrm{AD}$ as the main predictor. We started with a no change-point model and then tested a one-change-point model and finally a two change-points model. The two-change-point model function is given by

$Y_{i j}=\beta_{0}+b_{0 i}+\beta_{1}$ time $_{i j}+\beta_{2}\left(\right.$ time $_{i j}-$ $\left.c_{1}\right)_{+}+\beta_{3}\left(\text { time }_{i j}-c_{2}\right)_{+}+\varepsilon_{i j}$

where $(x)_{+}=x, x>0$ and $(x)_{+}=0, x<0$.

$c_{1}$ is first change-point, $c_{2}$ is second changepoint. $b_{0 i}$ is a random effect that follows a normal distribution with mean 0 and standard deviation of $\sigma$.

All models included baseline age, sex (male vs. female), race (white vs. non-white), and years of education as covariates. Model selections were based on the likelihood ratio 
Table 2: Fit statistics for models using no change-points, one change-point, or two change points. Model fit was assessed using the likelihood ratio test, with bold text indicating the current model provides significantly better fit compared to the one prior.

\begin{tabular}{|c|c|c|c|c|}
\hline & & $\begin{array}{l}\text { No change- } \\
\text { points }\end{array}$ & $\begin{array}{l}1 \text { change- } \\
\text { point }\end{array}$ & $\begin{array}{l}2 \text { change- } \\
\text { points }\end{array}$ \\
\hline \multirow[t]{2}{*}{ CVLT-IMM } & $-2 \mathrm{LL} / \mathrm{DF}$ & $6918.5 / 8$ & $6745.4 / 10$ & $6720.9 / 12$ \\
\hline & $P$ value & & $<.0001$ & $<.0001$ \\
\hline \multirow[t]{2}{*}{ CVLT-LD } & $-2 \mathrm{LL} / \mathrm{DF}$ & $4629.7 / 8$ & $4436.5 / 10$ & $4428.9 / 12$ \\
\hline & $\mathrm{P}$ value & & $<.0001$ & 0.022 \\
\hline \multirow[t]{2}{*}{ BVRT } & $-2 \mathrm{LL} / \mathrm{DF}$ & $5081.5 / 8$ & $5013.0 / 10$ & $5011.5 / 12$ \\
\hline & $P$ value & & $<.0001$ & 0.47 \\
\hline \multirow[t]{2}{*}{ TMT-A } & $-2 \mathrm{LL} / \mathrm{DF}$ & $8405.4 / 8$ & $8302.8 / 10$ & $8299.1 / 12$ \\
\hline & $\mathrm{P}$ value & & $<.0001$ & 0.16 \\
\hline \multirow[t]{2}{*}{ TMT-B } & $-2 \mathrm{LL} / \mathrm{DF}$ & $8701.8 / 8$ & $8626.8 / 10$ & $8626.5 / 12$ \\
\hline & $P$ value & & $<.0001$ & 0.86 \\
\hline \multirow[t]{2}{*}{ Categories } & $-2 \mathrm{LL} / \mathrm{DF}$ & $4245.0 / 8$ & $4073.8 / 10$ & $4064.1 / 12$ \\
\hline & $P$ value & & $<.0001$ & 0.0078 \\
\hline \multirow[t]{2}{*}{ Letters } & $-2 \mathrm{LL} / \mathrm{DF}$ & $4582.4 / 8$ & $4530.4 / 10$ & $4523.9 / 12$ \\
\hline & $\mathrm{P}$ value & & $<.0001$ & 0.039 \\
\hline \multirow[t]{2}{*}{ BNT } & $-2 \mathrm{LL} / \mathrm{DF}$ & $5152.7 / 8$ & $4963.6 / 10$ & 4949.9/12 \\
\hline & $\mathrm{P}$ value & & $<.0001$ & 0.0011 \\
\hline \multirow[t]{2}{*}{ Similarities } & $-2 \mathrm{LL} / \mathrm{DF}$ & $4221.8 / 8$ & $4154.9 / 10$ & 4147.3/12 \\
\hline & $\mathrm{P}$ value & & $<.0001$ & 0.022 \\
\hline \multirow[t]{2}{*}{ Card Rotations } & $-2 \mathrm{LL} / \mathrm{DF}$ & $7548.6 / 8$ & $7468.6 / 10$ & 7457.6/12 \\
\hline & $\mathrm{P}$ value & & $<.0001$ & 0.0041 \\
\hline \multirow[t]{2}{*}{ MMSE } & $-2 \mathrm{LL} / \mathrm{DF}$ & $3924.1 / 8$ & $3726.4 / 10$ & 3719.0/12 \\
\hline & $\mathrm{P}$ value & & $<.0001$ & 0.025 \\
\hline
\end{tabular}

Abbreviations: BNT, Boston Naming Test; BVRT, Benton Visual Retention Test; CVLT, California Verbal Learning Test; CVLT-IMM, CVLT immediate free recall; CVLT-LD, CVLT long delayed free recall; MMSE, Mini Mental State Exam, TMTA, Trail-Making Test-A, TMT-B, Trail-Making Test-B; -2LL/DF, likelihood ratio.

test. The best model fit tells us how many change-points, if any, there are for each cognitive measure.

The final model estimates were determined by a bootstrapping method so that the parameter estimates, and standard errors can be accurately captured, and more importantly, the timings of change-points from different cognitive measures can be statistically compared. We used 500 randomly resampled samples, with each sample having the same sample size as the original one for the bootstrapping. The models were fit using PROC NLMIXED in SAS 9.4 (Cary, NC). 
Table 3: Estimated change-points in years from AD diagnosis and slopes in test units change per year. All estimates, standard errors, and $\mathrm{p}$ values are from bootstrapping.

\begin{tabular}{|c|c|c|c|c|c|}
\hline Estimate (S.E.) & Change-point 1 & Change-point 2 & Slope 1 & Slope 2 & Slope 3 \\
\hline CVLT-IMM & $-11.65(0.80)$ & $-2.80(0.78)$ & $\begin{array}{c}0.23(0.28) \\
P=0.41\end{array}$ & $\begin{array}{c}-1.44(0.25) \\
\mathrm{P}<.0001\end{array}$ & $\begin{array}{c}-3.74(0.48) \\
\mathrm{P}<.0001\end{array}$ \\
\hline CVLT-LD & $-7.58(0.49)$ & $-4.21(0.33)$ & $\begin{array}{c}-0.080(0.041) \\
P=0.051\end{array}$ & $\begin{array}{c}-0.48(0.13) \\
P=0.0002\end{array}$ & $\begin{array}{c}-1.00(0.091) \\
P<.0001\end{array}$ \\
\hline $\mathrm{BVRT}^{*}$ & $-4.83(0.81)$ & NA & $\begin{array}{c}0.36(0.034) \\
\mathrm{P}<.0001\end{array}$ & $\begin{array}{c}0.99(0.12) \\
\mathrm{P}<.0001\end{array}$ & NA \\
\hline TMT-A* & $-1.90(0.68)$ & NA & $\begin{array}{l}0.59(0.14) \\
\mathrm{P}<.0001\end{array}$ & $\begin{array}{l}9.01(3.12) \\
P=0.0039\end{array}$ & NA \\
\hline TMT-B* & $-4.82(0.73)$ & NA & $\begin{array}{l}1.68(0.42) \\
\mathrm{P}<.0001\end{array}$ & $\begin{array}{c}11.86(2.21) \\
\mathrm{P}<.0001\end{array}$ & NA \\
\hline Categories & $-9.89(0.90)$ & $-3.17(0.41)$ & $\begin{array}{c}-0.13(0.035) \\
P=0.0002\end{array}$ & $\begin{array}{c}-0.34(0.052) \\
\mathrm{P}<.0001\end{array}$ & $\begin{array}{c}-0.97(0.095) \\
\mathrm{P}<.0001\end{array}$ \\
\hline Letters & $-10.03(1.39)$ & $-2.25(0.91)$ & $\begin{array}{c}-0.010(0.047) \\
\mathrm{P}=0.83\end{array}$ & $\begin{array}{c}-0.22(0.058) \\
P=0.00016\end{array}$ & $\begin{array}{l}-0.69(0.20) \\
P=0.00048\end{array}$ \\
\hline BNT & $-6.04(0.74)$ & $-1.51(0.57)$ & $\begin{array}{c}-0.10(0.036) \\
P=0.0043\end{array}$ & $\begin{array}{c}-0.89(0.17) \\
P<.0001\end{array}$ & $\begin{array}{l}-2.06(0.57) \\
P=0.00033\end{array}$ \\
\hline Similarities & $-10.65(1.24)$ & $-1.72(0.53)$ & $\begin{array}{c}0.038(0.054) \\
\mathrm{P}=0.48\end{array}$ & $\begin{array}{c}-0.16(0.043) \\
P=0.00026\end{array}$ & $\begin{array}{l}-0.84(0.23) \\
\mathrm{P}=0.00019\end{array}$ \\
\hline Card Rotation & $-15.48(1.72)$ & $-4.33(1.18)$ & $\begin{array}{c}1.66(1.59) \\
P=0.30\end{array}$ & $\begin{array}{c}-1.38(0.35) \\
\mathrm{P}<.0001\end{array}$ & $\begin{array}{c}-4.74(1.08) \\
P<.0001\end{array}$ \\
\hline MMSE & $-9.13(0.92)$ & $-1.77(0.44)$ & $\begin{array}{c}-0.038(0.024) \\
P=0.11\end{array}$ & $\begin{array}{c}-0.18(0.033) \\
P<.0001\end{array}$ & $\begin{array}{c}-1.03(0.15) \\
P<.0001\end{array}$ \\
\hline
\end{tabular}

* These measures only had a single change-point. Abbreviations: : BNT, Boston Naming Test; BVRT, Benton Visual Retention Test; CVLT, California Verbal Learning Test; CVLT-IMM, CVLT immediate free recall; CVLT-LD, CVLT long delayed free recall; MMSE, Mini Mental State Exam, TMT-A, Trail-Making Test-A; TMT-B, Trail-Making Test-B; S.E., standard error.

\section{Results}

Sample characteristics are shown in Table 1. The sample consisted of 165 participants with an AD diagnosis with a total of 988 visits. Average baseline age was 76.5 years (standard deviation, $[\mathrm{SD}]=7.4$ ), the average follow-up interval was 8.3 years $(\mathrm{SD}=6.0$, range $=0-24.1$, and average age at $\mathrm{AD}$ diagnosis was 86.5 years $(\mathrm{SD}=6.1)$. Eighty-three (50.3\%) participants were female, and $85.5 \%$ of the sample were white. The average years of education was 16.7 years $(\mathrm{SD}=2.6$, range $=8-21$ ).

\section{Change-point model comparisons for each} cognitive measure

Table 2 contains the results of the model fit statistics (likelihood ratio test) comparing the fit of three models for each cognitive measure. Models with 1-change-point provided better fit compared with no change-point models for all cognitive measures. Models with 2-change-points provided a better fit for CVLT-IMM, CVLT-LD, Category Fluency, Letter Fluency, BNT, Similarities, Card Rotations, and the MMSE. However, for the BVRT, TMT-A, and TMT-B, the 2-changepoint model did not significantly improve the 
This article is a US Government work. It is not subject to copyright under 17 USC 105 and is also made available for use under a CCO license.
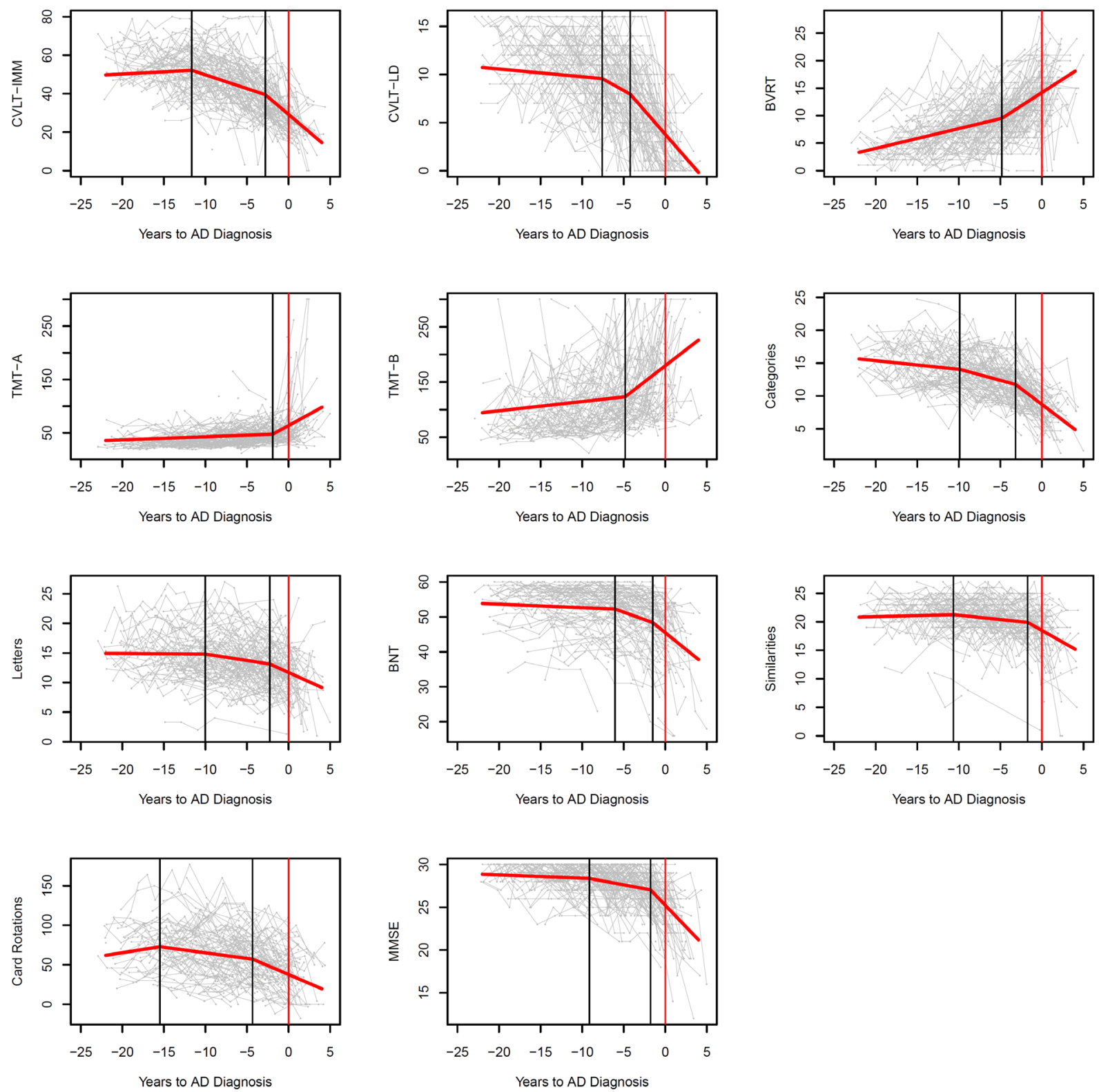

Figure 1: Line graphs showing the modelled population-level longitudinal trajectories from change-point models (in red) superimposed over spaghetti plots of the raw data (gray) for each cognitive measure. Vertical black lines indicate the change-points and the vertical red lines indicate timing of $\mathrm{AD}$ diagnosis. The $\mathrm{X}$-axis represents years before $\mathrm{AD}$ diagnosis. Measurement units: CVLT-IMM, total correct out of 80; CVLT-LD, total correct out of 16; BVRT, total number of errors; TMT-A, seconds to complete; TMT-B, seconds to complete; Categories, mean number correct words; Letters, mean number correct words; BNT, correct out of 60; Similarities, total our of 28; Card Rotations, total out of 224; MMSE total out of 30. 
model fit, indicating a 1-change-point model was the best fitting model for these measures.

\section{Temporal position of change-points and subsequent rates of decline}

Table 3 shows the results of parameter estimates from bootstrapping the best fitting model for each cognitive measure, including the estimated change-points, the rate of

change at each segment of the trajectory, and corresponding standard errors (SE). Figure 1 shows the estimated trajectories superimposed over the raw data for each cognitive measure.

\section{Learning and memory}

CVLT-IMM: The estimated first changepoint was $11.65(\mathrm{SE}=0.80)$ years before $\mathrm{AD}$ diagnosis, when the trajectory transitioned from non-significant increase in performance over time $(0.23$ items per year, $\mathrm{SE}=0.28)$ to significant moderate decline in performance $(-1.44$ items per year, $\mathrm{SE}=0.25)$. The second change-point was $2.80(\mathrm{SE}=0.78)$ years before $\mathrm{AD}$ diagnosis, when decline accelerated again $(-3.74$ items per year, $\mathrm{SE}=0.48)$.

CVLT-LD: The estimated first change-point was $7.58(\mathrm{SE}=0.49)$ years before $\mathrm{AD}$ diagnosis, when the trajectory transitioned from trending-significant minor decrease in performance over time $(-0.080$ items per year, $\mathrm{SE}=0.041)$ to significant moderate decline in performance $(-0.48$ items per year, $\mathrm{SE}=0.13)$. The second change-point was $4.21(\mathrm{SE}=0.34)$ years before AD diagnosis, when decline accelerated again $(-1.00$ items per year, $\mathrm{SE}=0.091$ ).

BVRT: The estimated change-point was 4.83 $(\mathrm{SE}=0.81)$ years before $\mathrm{AD}$ diagnosis, when the trajectory transitioned from significant modest decline in performance over time ( 0.36 errors per year, $\mathrm{SE}=0.034)$ to significant accelerated decline in performance (0.99 errors per year, $\mathrm{SE}=0.12$ ).

\section{Attention and executive function}

TMT-A: The estimated change-point was $1.90(\mathrm{SE}=0.68)$ years before $\mathrm{AD}$ diagnosis, when the trajectory transitioned from significant modest decline in performance over time (0.59 seconds per year, $\mathrm{SE}=0.14)$ to significant accelerated decline in performance (9.01 seconds per year, $\mathrm{SE}=3.12$ ).

TMT-B: The estimated change-point was $4.82(\mathrm{SE}=0.73)$ years before $\mathrm{AD}$ diagnosis, when the trajectory transitioned from significant modest decline in performance over time (1.68 seconds per year, $\mathrm{SE}=0.42$ ) to significant accelerated decline in performance (11.86 seconds per year, $\mathrm{SE}=2.21$ ).

\section{Verbal fluency}

Category fluency: The estimated first change-point was 9.89 ( $\mathrm{SE}=0.90)$ years before $\mathrm{AD}$ diagnosis, when the trajectory transitioned from significant minor decrease in performance over time ( -0.13 words per year, $\mathrm{SE}=0.035)$ to significant moderate decline in performance $(-0.34$ words per year, $\mathrm{SE}=$ 0.052). The second change-point was 3.17 $(\mathrm{SE}=0.41)$ years before $\mathrm{AD}$ diagnosis, when decline accelerated again ( -0.97 words per year, $\mathrm{SE}=0.095$ ).

Letter fluency: The estimated first changepoint was $10.03(\mathrm{SE}=1.39)$ years before $\mathrm{AD}$ diagnosis, when the trajectory transitioned from non-significant minor decrease in performance over time $(-0.010$ words per year, $\mathrm{SE}=0.047)$ to significant moderate decline in performance $(-0.22$ words per year, $\mathrm{SE}=$ 0.058). The second change-point was 2.25 $(\mathrm{SE}=0.91)$ years before $\mathrm{AD}$ diagnosis, when decline accelerated again $(-0.69$ words per year, $\mathrm{SE}=0.20$ ).

\section{Object recognition and naming}

BNT: The estimated first change-point was $6.04(\mathrm{SE}=0.74)$ years before $\mathrm{AD}$ diagnosis, when the trajectory transitioned from significant minor decrease in performance over time $(-0.10$ words per year, $\mathrm{SE}=0.036)$ to 
significant moderate decline in performance $(-0.89$ words per year, $\mathrm{SE}=0.17)$. The second change-point was $1.51(\mathrm{SE}=0.57)$ years before $\mathrm{AD}$ diagnosis, when decline accelerated again $(-2.06$ words per year, $\mathrm{SE}=0.57)$.

\section{Abstract reasoning}

Similarities: The estimated first change-point was $10.65(\mathrm{SE}=1.24)$ years before $\mathrm{AD}$ diagnosis, when the trajectory transitioned from non-significant increase in performance over time $(0.038$ points per year, $\mathrm{SE}=0.054)$ to significant moderate decline in performance (0.16 points per year, $\mathrm{SE}=0.043)$. The second change-point was $1.72(\mathrm{SE}=0.35)$ years before $\mathrm{AD}$ diagnosis, when decline accelerated again $(-0.84$ points per year, $\mathrm{SE}=0.23)$.

\section{Visuospatial ability}

Card Rotations: The estimated first changepoint was $15.48(\mathrm{SE}=1.72)$ years before $\mathrm{AD}$ diagnosis, when the trajectory transitioned from non-significant increase in performance over time (1.66 points per year, $\mathrm{SE}=1.59)$ to significant moderate decline in performance $(-1.38$ points per year, $\mathrm{SE}=0.35)$. The second change-point was $4.33(\mathrm{SE}=1.18)$ years before $\mathrm{AD}$ diagnosis, when decline accelerated again $(-4.74$ points per year, $\mathrm{SE}=1.08)$.

\section{Global Cognitive Performance}

MMSE: The estimated first change-point was $9.13(\mathrm{SE}=0.92)$ years before $\mathrm{AD}$ diagnosis, when the trajectory transitioned from nonsignificant increase in performance over time $(-0.038$ units per year, $\mathrm{SE}=0.024)$ to significant moderate decline in performance $(-0.18$ units per year, $\mathrm{SE}=0.033)$. The second change-point was $1.77(\mathrm{SE}=0.47)$ years before $\mathrm{AD}$ diagnosis, when decline accelerated again $(-1.03$ units per year, $\mathrm{SE}=0.15)$.

\section{Comparing change-points across cognitive measures}

Figure 2 provides a schematic overview of estimated change-points for each cognitive measure. Table 4 shows the results from using bootstrapping to compare the first change-points for each measure against all other measures to identify the earliest changing measures. The measure with the earliest change-point was Card Rotations, which was significantly earlier than all other measures. The next measure to show an early changepoint was CVLT-IMM, which was significantly earlier than CVLT-LD, BVRT, TMTA, TMT-B, BNT, and MMSE but not significantly earlier than measures of verbal fluency, or Similarities. The measure to show the latest change-point in relation to AD diagnosis was TMT-A. Supplemental Table 1 shows the results from using bootstrapping to compare the second change-points for each measure. The second change-point for Card Rotations was not significantly earlier than the second change-points for CVLT measures.

\section{Discussion}

In a sample of participants with consensus diagnoses of clinical AD, we used extensive longitudinal cognitive data to examine the temporal sequence of stages of decline in 11 cognitive measures. Change-points identifying steeper rates of cognitive decline ranged from 15.5 years before AD diagnosis for the Card Rotations test to 1.9 years before AD diagnosis for TMT-A. While episodic memory assessed by CVLT measures was not the domain to show the earliest changes in rates of decline, changes were still detected up to 11.7 years before AD diagnosis. Using changepoint analyses in this way can reveal the temporal ordering of domain-specific accelerated decline in preclinical AD.

The change-point for Card Rotations (15.5 years before AD diagnosis) was significantly earlier than change-points for all other cognitive measures, including CVLT measures of episodic memory. This extends previous findings from a systematic review [14] in which it was casually observed that 
medRxiv preprint doi: https://doi.org/10.1101/19009696; this version posted October 22, 2019. The copyright holder for this preprint (which was not certified by peer review) is the author/funder, who has granted medRxiv a license to display the preprint in perpetuity.

This article is a US Government work. It is not subject to copyright under 17 USC 105 and is also made available for use under a CCO license.

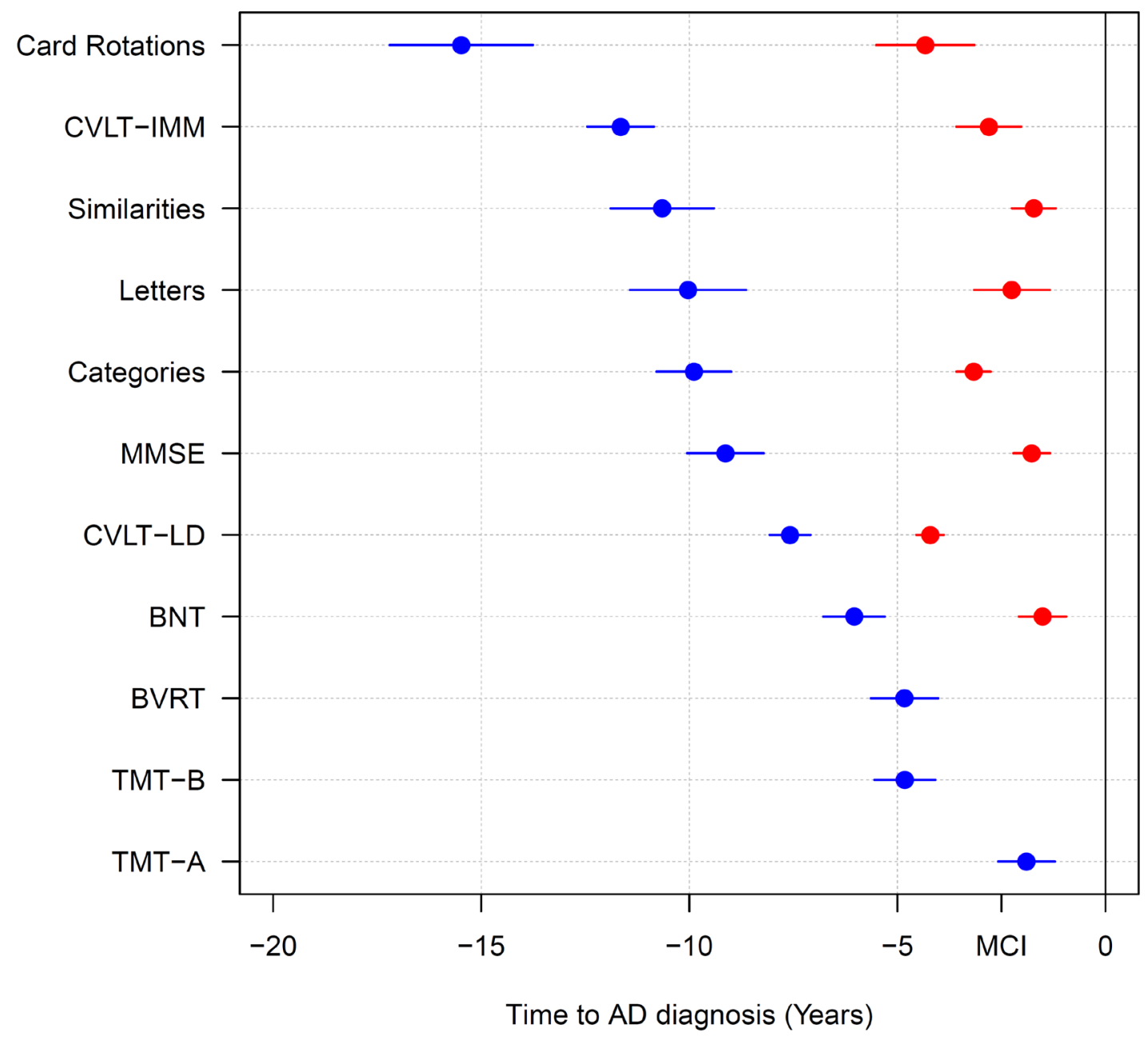

Figure 2: Dot plot comparing the estimated change points relative to AD diagnosis across cognitive measures. Cognitive measures are presented in order of first change-points. Red dots represent first change-points and blue points represent second change-points. Extended lines show standard errors. MCI indicates the average time of mild cognitive impairment symptom onset before AD diagnosis. 
Table 4: The first change-point for each measure was compared between all measures using bootstrapping. P-values are presented to show which measures were significantly different from each other.

\begin{tabular}{|c|c|c|c|c|c|c|c|c|c|c|c|}
\hline & $\begin{array}{l}\text { CVLT- } \\
\text { IMM }\end{array}$ & $\begin{array}{l}\text { CVLT- } \\
\text { LD }\end{array}$ & BVRT & TMT-A & TMT-B & Categories & Letters & $\mathrm{BNT}$ & Similarities & $\begin{array}{l}\text { Card } \\
\text { Rotation }\end{array}$ & MMSE \\
\hline CVLT-IMM & 1 & $<.0001$ & $<.0001$ & $<.0001$ & $<.0001$ & 0.13 & 0.31 & $<.0001$ & 0.46 & 0.045 & 0.031 \\
\hline CVLT-LD & & 1 & 0.0029 & $<.0001$ & 0.0022 & 0.031 & 0.10 & 0.087 & 0.025 & $<.0001$ & 0.13 \\
\hline TMT-A & & & & 1 & 0.00063 & $<.0001$ & $<.0001$ & $<.0001$ & $<.0001$ & $<.0001$ & $<.0001$ \\
\hline TMT-B & & & & & 1 & $<.0001$ & 0.00057 & 0.27 & $<.0001$ & $<.0001$ & 0.00034 \\
\hline Categories & & & & & & 1 & 0.93 & 0.0014 & 0.64 & 0.0041 & 0.56 \\
\hline $\mathrm{BNT}$ & & & & & & & & 1 & 0.0017 & $<.0001$ & 0.013 \\
\hline Similarities & & & & & & & & & 1 & 0.023 & 0.33 \\
\hline Card Rotation & & & & & & & & & & 1 & 0.0015 \\
\hline MMSE & & & & & & & & & & & 1 \\
\hline
\end{tabular}

Abbreviations: BNT, Boston Naming Test; BVRT, Benton Visual Retention Test; CVLT, California Verbal Learning Test; CVLT-IMM, CVLT immediate free recall; CVLT-LD, CVLT long delayed free recall; MMSE, Mini Mental State Exam, TMT-A, Trail-Making Test-A; TMT-B, Trail-Making Test-B. 
visuospatial ability shows the earliest acceleration of cognitive decline prior to AD.

The underlying mechanisms that may lead to early accelerated decline in visuospatial ability in preclinical AD may be understood from the roles of the precuneus and other parietal regions in visuospatial tasks that involve spatial manipulation (as is the case with the Card Rotations Task in BLSA). [32] The precuneus is also part of a large network that includes medial temporal lobe and frontal lobe regions that support spatial navigation. [33] The precuneus is one of the earliest brain regions to show accumulation of $\mathrm{A} \beta$ in preclinical AD $[34,35]$ and deficits in spatial navigation are one of the earliest impairments leading to loss of independence. Taken together, the functional importance of the precuneus in visuospatial processing and its susceptibility to early AD pathology support our finding that visuospatial ability would be affected early in preclinical AD.

CVLT-IMM showed the second earliest change-point at 11.7 years before diagnosis. This change was significantly earlier than CVLT-LD, which had a change-point at 7.6 years before AD diagnosis. The difference between change-points for CVLT-IMM and CVLT-LD is consistent with previous studies that reported faster rates of verbal learning compared to delayed free recall declines at earlier stages of disease progression, $[36,37]$ and confirms the importance of early learning deficits in detecting individuals at risk of developing AD. [38] However, change-points for CVLT-IMM were not significantly earlier than those for measures of verbal fluency, the Similarities Test, or the MMSE, suggesting that some aspects of executive function, i.e., verbal concept formation and abstract reasoning, as well as aspects of mental status may exhibit changes in the rates of decline as early as some memory-based learning tasks. These results contrast with earlier reports using BLSA data indicating that memory is affected earlier than executive function. [13] One possible explanation for the different pattern of results in the present analysis is the larger number of participants with longer follow-up compared to previous reports.

Every cognitive measure examined showed at least one change-point, with the majority of measures exhibiting two change-points. In measures with two change points, the first change-point was always more than five years before diagnosis while the second change-point was less than five years before diagnosis and was followed by even faster rates of decline than the first change-point. The second change-points appear to represent the transition from preclinical AD to MCI. The mean time between symptom onset of $\mathrm{MCI}$ and $\mathrm{AD}$ diagnosis in our sample was 2.5 $(\mathrm{SD}=1.8)$ years. Accelerated cognitive declines in the years immediately prior to symptom onset and $\mathrm{AD}$ diagnosis are consistent with other reports that MCI participants show raster rates of decline compared to healthy controls for a range of cognitive domains including memory, executive function, attention and verbal fluency. [39]

The temporal ordering of the first and second change-points across cognitive measures were similar. However, the second changepoint for Card Rotations was not significantly earlier than the second change-points for measures of memory or fluency, suggesting that there is little difference in the sensitivity of these measures as the time to diagnosis becomes shorter. As noted by Grober et al. [13] the temporal unfolding of cognitive decline identified by change-point studies implies that the predictive utility of different measures would be expected to vary by time from AD diagnosis. As such, the temporal ordering of change-points in the present study would suggest that measures of visuospatial ability and memory may serve as predictive tools for the development of AD as much as 15 years before diagnosis with other 
measures becoming more relevant closer to diagnosis. However, some measures of processing speed, i.e., TMT-A, may only have predictive utility less than five years before diagnosis, during a period when MCI may already be detectible. This interpretation is supported by previous reports of the predictive power of different cognitive measures. [40]

A limitation of this study is that BLSA participants are a highly educated group, which may limit generalizability. Additionally, the focus of this study was on defining and comparing population-level change-points and as such we did not examine individual differences in the timing of change-points which requires longer follow-up at the individual level. Future work is needed to assess individual differences in change-points. However, the strengths of this study are the comprehensive cognitive battery, frequent visit schedule (subjects were tested annually or biannually in this sample) and consensus-based determination of symptom onset and AD

diagnosis. In addition, the use of the bootstrapping analysis not only allows us to capture parameter estimates and standard errors more accurately, but enables the statistical comparison of change-points among different cognitive measures, greatly extending the work of previous studies. [14]

In summary, we found that the cognitive measure to show the earliest change in rates of decline in preclinical AD was visuospatial ability rather than episodic memory. Using change-point analyses with bootstrapping can reveal the temporal patterns of accelerated cognitive decline in preclinical $A D$ and may help guide the development of tools for participant screening in clinical trials.

\section{Acknowledgements}

This research was supported entirely by the Intramural Research Program of the National
Institutes of Health, National Institute on Aging. We thank the staff of the BLSA and LBN cognitive testing group for their assistance and the BLSA participants for their dedication to this study.

\section{Declaration of interest}

Declaration of interest: None.

\section{References}

[1] Sperling RA, Aisen PS, Beckett LA, Bennett DA, Craft S, Fagan AM, et al. Toward defining the preclinical stages of Alzheimer's disease: Recommendations from the National Institute on AgingAlzheimer's Association workgroups on diagnostic guidelines for Alzheimer's disease. Alzheimer's \& dementia. 2011;7:280-92.

[2] Bäckman L, Jones S, Berger A-K, Laukka EJ, Small BJ. Cognitive impairment in preclinical Alzheimer's disease: A metaanalysis. Neuropsychology. 2005;19:520-31. [3] Armstrong NM, Huang C-W, Williams OA, Bilgel M, An Y, Doshi J, et al. Sex differences in the association between amyloid and longitudinal brain volume change in cognitively normal older adults. NeuroImage: Clinical. 2019;22:101769.

[4] Jack Jr CR, Bennett DA, Blennow K, Carrillo MC, Dunn B, Haeberlein SB, et al. NIA-AA Research Framework: Toward a biological definition of Alzheimer's disease. Alzheimer's \& Dementia. 2018;14:535-62.

[5] Villemagne VL, Burnham S, Bourgeat P, Brown B, Ellis KA, Salvado O, et al. Amyloid $\beta$ deposition, neurodegeneration, and cognitive decline in sporadic Alzheimer's disease: a prospective cohort study. The Lancet Neurology. 2013;12:357-67.

[6] van Dyck $\mathrm{CH}$. Anti-amyloid- $\beta$ monoclonal antibodies for Alzheimer's disease: pitfalls and promise. Biological psychiatry. 2018;83:311-9. 
[7] Sarazin M, Dorothée G, de Souza LC, Aucouturier P. Immunotherapy in Alzheimer's disease: do we have all the pieces of the puzzle? Biological Psychiatry. 2013;74:329-32.

[8] Sevigny J, Chiao P, Bussière T, Weinreb PH, Williams L, Maier M, et al. The antibody aducanumab reduces $A \beta$ plaques in Alzheimer's disease. Nature. 2016;537:50.

[9] Sperling RA, Rentz DM, Johnson KA, Karlawish J, Donohue M, Salmon DP, et al. The A4 study: stopping AD before symptoms begin? Science translational medicine. 2014; 6:228fs $13-\mathrm{fs} 13$.

[10] Hall CB, Ying J, Kuo L, Lipton RB. Bayesian and profile likelihood change point methods for modeling cognitive function over time. Computational Statistics \& Data Analysis. 2003;42:91-109.

[11] Hall CB, Ying J, Kuo L, Sliwinski M, Buschke H, Katz M, et al. Estimation of bivariate measurements having different change points, with application to cognitive ageing. Statistics in Medicine. 2001;20:3695714.

[12] Grober E, An Y, Lipton RB, Kawas C, Resnick SMJJotINS. Timing of onset and rate of decline in learning and retention in the pre-dementia phase of Alzheimer's disease. 2019:1-7.

[13] Grober E, Hall CB, Lipton RB, Zonderman AB, Resnick SM, Kawas C. Memory impairment, executive dysfunction, and intellectual decline in preclinical Alzheimer's disease. Journal of the International Neuropsychological Society. 2008;14:266-78.

[14] Karr JE, Graham RB, Hofer SM, MunizTerrera G. When does cognitive decline begin? A systematic review of change point studies on accelerated decline in cognitive and neurological outcomes preceding mild cognitive impairment, dementia, and death. Psychology and aging. 2018;33:195.

[15] Thorvaldsson V, MacDonald SW, Fratiglioni L, Winblad B, Kivipelto M,
Laukka EJ, et al. Onset and rate of cognitive change before dementia diagnosis: findings from two Swedish population-based longitudinal studies. Journal of the International Neuropsychological Society. 2011;17:154-62.

[16] Laukka EJ, MacDonald SWS, Fratiglioni L, Bäckman L. Preclinical Cognitive Trajectories Differ for Alzheimer's Disease and Vascular Dementia. Journal of the International Neuropsychological Society. 2012;18:191-9.

[17] Shock NW. Normal human aging: The Baltimore longitudinal study of aging. 1984. [18] Morris JC. The Clinical Dementia Rating (CDR): current version and scoring rules. Neurology. 1993.

[19] Fuld PA. Psychological testing in the differential diagnosis of the dementias. Alzheimer's disease: senile dementia and related disorders. 1978;7:185-93.

[20] Petersen RC. Mild cognitive impairment as a diagnostic entity. Journal of internal medicine. 2004;256:183-94.

[21] Association AP. Diagnostic and Statistical Manual of Mental Health Disorders (DSM-III-R): American Psychiatric Association; 1987.

[22] McKhann G, Drachman D, Folstein M, Katzman R, Price D, Stadlan EM. Clinical diagnosis of Alzheimer's disease Report of the NINCDS-ADRDA Work Group* under the auspices of Department of Health and Human Services Task Force on Alzheimer's Disease. Neurology. 1984;34:939-.

[23] Delis DC, Kramer JH, Kaplan E, Thompkins BAO. CVLT: California verbal learning test-adult version: manual: Psychological Corporation; 1987.

[24] Benton AL. Visual retention test. New York, NY: Psychological Corporation; 1974. [25] Reitan RM. Trail Making Test: Manual for administration and scoring: Reitan Neuropsychology Laboratory; 1992. 
[26] Benton AL. Differential behavioral effects in frontal lobe disease. Neuropsychologia. 1968;6:53-60.

[27] Newcombe F. Missile wounds of the brain: A study of psychological deficits. 1969.

[28] Kaplan E, Goodglass H, Weintraub S. The Boston Naming Test (2nd ed.). Philadelphia, PA: Lea \& Febiger; 1983.

[29] Wechsler D. WAIS-R manual: Wechsler adult intelligence scale-revised:

Psychological Corporation; 1981.

[30] Wilson JR, De Fries J, Mc Clearn G, Vandenberg S, Johnson R, Rashad M. Cognitive abilities: Use of family data as a control to assess sex and age differences in two ethnic groups. The International Journal of Aging and Human Development. 1975;6:261-76.

[31] Folstein MF, Folstein SE, McHugh PRJJopr. "Mini-mental state": a practical method for grading the cognitive state of patients for the clinician. 1975;12:189-98.

[32] Cavanna AE, Trimble MRJB. The precuneus: a review of its functional anatomy and behavioural correlates. 2006;129:56483.

[33] Coughlan G, Laczó J, Hort J, Minihane A-M, Hornberger MJNRN. Spatial navigation deficits-overlooked cognitive marker for preclinical Alzheimer disease? 2018; 14:496.

[34] Bilgel M, Prince JL, Wong DF, Resnick SM, Jedynak BMJN. A multivariate nonlinear mixed effects model for longitudinal image analysis: Application to amyloid imaging. 2016;134:658-70.

[35] Rodrigue K, Kennedy K, Devous M, Rieck J, Hebrank A, Diaz-Arrastia R, et al. $\beta$ Amyloid burden in healthy aging: regional distribution and cognitive consequences. 2012;78:387-95.

[36] Bilgel M, An Y, Lang A, Prince J, Ferrucci L, Jedynak B, et al. Trajectories of Alzheimer disease-related cognitive measures in a longitudinal sample. 2014; 10:735-42. e4.

[37] Grober E, Kawas CJP, aging. Learning and retention in preclinical and early Alzheimer's disease. 1997;12:183. [38] Chang Y-L, Bondi MW, FennemaNotestine C, McEvoy LK, Hagler Jr DJ, Jacobson MW, et al. Brain substrates of learning and retention in mild cognitive impairment diagnosis and progression to Alzheimer's disease. 2010;48:1237-47. [39] Johnson JK, Gross AL, Pa J, McLaren DG, Park LQ, Manly JJ, et al. Longitudinal change in neuropsychological performance using latent growth models: a study of mild cognitive impairment. 2012;6:540-50.

[40] Saxton J, Lopez OL, Ratcliff G, Dulberg C, Fried LP, Carlson MC, et al. Preclinical Alzheimer disease: neuropsychological test performance 1.5 to 8 years prior to onset. 2004;63:2341-7. 